\title{
On the minimum size of hamiltonian saturated hypergraphs
}

\author{
Andrzej Ruciński* \\ Department of Discrete Mathematics \\ Adam Mickiewicz University \\ Poznań, Poland \\ rucinski@amu.edu.pl
}

\author{
Andrzej Żak ${ }^{\dagger}$ \\ Faculty of Applied Mathematics \\ AGH University of Science and Technology \\ Kraków, Poland \\ zakandrz@agh.edu.pl
}

Submitted: Dec 27, 2018; Accepted: Nov 3, 2020; Published: Nov 27, 2020

(C) The authors. Released under the CC BY-ND license (International 4.0).

\begin{abstract}
For $1 \leqslant \ell<k$, an $\ell$-overlapping $k$-cycle is a $k$-uniform hypergraph in which, for some cyclic vertex ordering, every edge consists of $k$ consecutive vertices and every two consecutive edges share exactly $\ell$ vertices. A $k$-uniform hypergraph $H$ is $\ell$-hamiltonian saturated if $H$ does not contain an $\ell$-overlapping hamiltonian $k$ cycle but every hypergraph obtained from $H$ by adding one edge does contain such a cycle. Let $\operatorname{sat}(N, k, \ell)$ be the smallest number of edges in an $\ell$-hamiltonian saturated $k$-uniform hypergraph on $N$ vertices. In the case of graphs Clark and Entringer showed in 1983 that $\operatorname{sat}(N, 2,1)=\left\lceil\frac{3 N}{2}\right\rceil$. The present authors proved that for $k \geqslant 3$ and $\ell=1$, as well as for all $0.8 k \leqslant \ell \leqslant k-1$, $\operatorname{sat}(N, k, \ell)=\Theta\left(N^{\ell}\right)$. Here we prove that $\operatorname{sat}(N, 2 \ell, \ell)=\Theta\left(N^{\ell}\right)$.
\end{abstract}

Mathematics Subject Classifications: 05C65

\section{Introduction}

A $k$-uniform hypergraph ( $k$-graph for short) is a pair $H=(V, E)$, where $V$ is a finite set (of vertices) and $E \subseteq\left(\begin{array}{l}V \\ k\end{array}\right)$ is a family of $k$-element subsets of $V$ called edges of $H$. We will often identify $H$ with its vertex set $E$. For instance, we will denote by $|H|$ the number of edges in $H$.

Given integers $1 \leqslant \ell<k$, we define an $\ell$-overlapping $k$-cycle or, shortly, $(\ell, k)$-cycle, as a $k$-graph in which, for some cyclic ordering of its vertices, every edge consists of $k$ consecutive vertices, and every two consecutive edges (in the natural ordering of the

${ }^{*}$ Research supported by the Polish NSC grants 2014/15/B/ST1/01688 and 2018/29/B/ST1/00426.

${ }^{\dagger}$ Research partially supported by the Polish Ministry of Science and Higher Education. 
edges induced by the ordering of the vertices) share exactly $\ell$ vertices. An $\ell$-overlapping $k$-path (or $(\ell, k)$-path) is defined similarly, that is, with vertices ordered $v_{1}, \ldots, v_{s}$, the edges of the path are $\left\{v_{1}, \ldots, v_{k}\right\},\left\{v_{k-\ell+1}, \ldots, v_{k+\ell}\right\}, \ldots,\left\{v_{s-k+1}, \ldots, v_{s}\right\}$. Note that the number of edges of an $(\ell, k)$-cycle with $s$ vertices is $s /(k-\ell)$ (and thus, $s$ is divisible by $k-\ell$ ). Likewise, it can be easily seen that the number of vertices of an $(\ell, k)$-path equals $\ell$ modulo $k-\ell$.

Given a $k$-graph $H$ and a $k$-element set $e \in H^{c}$, where $H^{c}=\left(\begin{array}{c}V \\ k\end{array}\right) \backslash H$ is the complement of $H$, we denote by $H+e$ the hypergraph obtained from $H$ by adding $e$ to its edge set. For $1 \leqslant \ell \leqslant k-1$, a $k$-graph $H$ is $\ell$-hamiltonian saturated (a.k.a. maximally non- $\ell$ hamiltonian) if $H$ is not $\ell$-hamiltonian but for every $e \in H^{c}$ the $k$-graph $H+e$ is such. The largest number of edges in an $\ell$-hamiltonian saturated $k$-graph on $N$ vertices has been determined in [5].

In this paper we are interested in the other extreme. For $N$ divisible by $k-\ell$, let $\operatorname{sat}(N, k, \ell)$ be the smallest number of edges in an $\ell$-hamiltonian saturated $k$-graph on $N$ vertices. In the case of graphs, Clark and Entringer proved in 1983 that

$$
\operatorname{sat}(N, 2,1)=\left\lceil\frac{3 N}{2}\right\rceil \text { for } N \geqslant 52 .
$$

For $k$-graphs with $k \geqslant 3$ the problem was first mentioned in $[6,7]$. It seems to be quite hard to obtain such precise results as for graphs. Therefore, the emphasis has been put on the order of magnitude of $\operatorname{sat}(N, k, \ell)$. It is quite easy to see that

$$
\operatorname{sat}(N, k, \ell)=\Omega\left(N^{\ell}\right) \text {, for all } k \geqslant 3,1 \leqslant \ell \leqslant k-1,
$$

(see, e.g., Proposition 2.1 in [8]). The present authors proved in [8] that for $k \geqslant 3$ and $\ell=1$, as well as for all $0.8 k \leqslant \ell \leqslant k-1$,

$$
\operatorname{sat}(N, k, \ell)=\Theta\left(N^{\ell}\right)
$$

(see [10] for the case $\ell=k-1$ ). We also conjectured that (3) holds true for all $1 \leqslant \ell \leqslant k-1$. In [9] we proved a weaker general upper bound

$$
\operatorname{sat}(N, k, \ell)=O\left(N^{\frac{k+\ell}{2}}\right) .
$$

In the same paper we improved the above bound in the smallest open case by showing that $\operatorname{sat}(N, 4,2)=O\left(N^{\frac{14}{5}}\right)$. In this paper we confirm our conjecture in the middle of the range.

Theorem 1. For all $\ell \geqslant 2$ and $N$ divisible by $\ell, \operatorname{sat}(N, 2 \ell, \ell)=\Theta\left(N^{\ell}\right)$.

Our proof combines two general approaches to this type of problems developed, respectively, in [8] and [10,9].

\section{Construction}

In this section, after setting some parameters, we will describe our construction and present the proof of Theorem 1 based on two lemmas which will be proved later. 


\subsection{Parameters setting}

We need to choose the values of some parameters carefully and in doing so a pivotal role is played by the following notion. Given a positive integer $x$, let $C$ and $D$ be two disjoint sets with $|C|=x$ and $|D|=\infty$. Let $\nu(x)=\max _{P}|V(P)|$, where the maximum is taken over all $(\ell, 2 \ell)$-paths $P$ which are subgraphs of the complete $2 \ell$-uniform hypergraph with vertex set $C \cup D$ and such that

$$
C \subset V(P) \subset C \cup D \quad \text { and } \quad|e \cap C| \geqslant \ell+1 \quad \text { for all } \quad e \in P .
$$

Proposition 2. If $x \geqslant \ell+1$, then

$$
\nu(x)= \begin{cases}x \frac{2 \ell}{\ell+1}, & \text { if }(\ell+1) \mid x, \\ \left\lfloor\frac{x}{\ell+1}\right\rfloor 2 \ell+\ell, & \text { otherwise. }\end{cases}
$$

In particular,

$$
\nu(x) \geqslant \frac{2 \ell}{\ell+1} x-\ell
$$

Proof. Let $x=q(\ell+1)+r$, where $q=\left\lfloor\frac{x}{\ell+1}\right\rfloor$ and $0 \leqslant r \leqslant \ell$. Let $P$ be an $(\ell, 2 \ell)$-path with $|V(P)|=\nu(x)$ and $t$ edges satisfying (4). Let $e_{1}, \ldots, e_{t}$ be the edges of $P$ in the linear order underlying $P$. Set $s=\left\lfloor\frac{t+1}{2}\right\rfloor$. Clearly, $t \in\{2 s-1,2 s\}$. Recall that, by (4), $\left|e_{i} \cap C\right| \geqslant \ell+1$ for each $i \in\{1, \ldots, 2 s-1\}$. Hence, $s \leqslant q$, because $e_{1}, e_{3}, \ldots, e_{2 s-1}$ are pairwise disjoint. Also by (4), if $t=2 s$, then

$$
\left(e_{t} \cap C\right) \backslash \bigcup_{j=1}^{s} e_{2 j-1}=\left(e_{t} \cap C\right) \backslash e_{2 s-1} \neq \emptyset .
$$

Thus, if $r=0$, then $t=2 s-1$ and $|V(P)|=s \cdot 2 \ell$. Otherwise, $t \leqslant 2 s$ and $|V(P)| \leqslant s \cdot 2 \ell+\ell$, and so the right-hand-side of (5) is the upper bound on $|V(P)|$.

To show equality in (5), let us view $P$ as a binary sequence $Q$, where each vertex of $C$ is represented by a symbol $c$ and each vertex of $V(P) \cap D$ is represented by a symbol $d$. (And the edges of $P$ follow the sequence $Q$ according to the definition of an $(\ell, 2 \ell)$-path.) We now construct a sequence $Q$ which yields a path $P$ satisfying (4) and with $|V(P)|$ equal to the R-H-S of (5).

Let $Q$ begin with $\ell-1$ vertices from $D$ and then traverse a group of $\ell+1$ vertices from $C$, and so on $q$ times. If $r>0$, then at the end we add $r$ vertices from $C$ followed by $\ell-r$ vertices from $D$ (see (7) below).

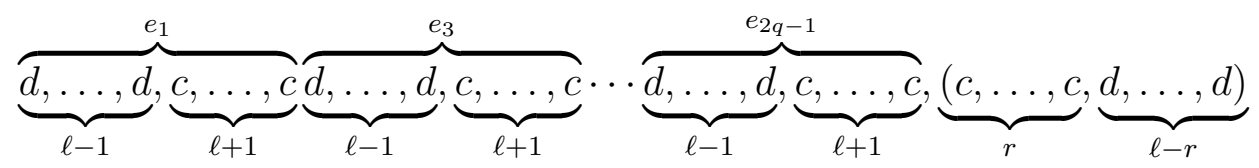

It is easy to check that $P$ satisfies (4). Clearly, $|V(P)|=q \cdot 2 \ell$, if $r=0$, and $|V(P)|=$ $q \cdot 2 \ell+\ell$, if $r>0$. 
The function $\nu(x)$ is non-decreasing, but, as an immediate consequence of Proposition 2 , it cannot increase too fast.

Proposition 3. For all $x \geqslant 1$ we have $\nu(x-1) \geqslant \nu(x)-\ell$. Moreover, if $x$ or $x-1$ is divisible by $\ell+1$, then $\nu(x-1)=\nu(x)-\ell$.

Proof. Let $x=q(\ell+1)+r$ as in the proof of Proposition 2. It is easy to check that, by (5), if $2 \leqslant r \leqslant \ell$, then $\nu(x-1)=\nu(x)$, while in the remaining two cases, $r=0$ and $r=1$, we have $\nu(x-1)=\nu(x)-\ell$.

We now define parameters and sets our construction will rely upon. Let

$$
N_{0}=100 \ell^{5}
$$

and let $N \geqslant N_{0}$ be an integer divisible by $\ell$. Define integers

$$
n=\left\lfloor\frac{N+4 \ell^{3}}{8 \ell^{3}+2 \ell}\right\rfloor
$$

and

$$
a=\frac{N+4 \ell^{3}-n\left(8 \ell^{3}+2 \ell\right)}{\ell} .
$$

Using (8), it is easy to check that

$$
n \geqslant 10 \ell^{2}
$$

Moreover, by (9), $n>\frac{N+4 \ell^{3}}{8 \ell^{3}+2 \ell}-1$, which is equivalent to $a<8 \ell^{2}+2$. Consequently, in view of (11), $a \leqslant n-1$. Let

$$
x_{i}= \begin{cases}4 \ell^{2}(\ell+1)+2 \ell+1, & i=1, \ldots, a, \\ 4 \ell^{2}(\ell+1)+2 \ell, & i=a+1, \ldots, n .\end{cases}
$$

Proposition 4. For each $I \subset\{1, \ldots, n\}$ with $|I|=n-1$

$$
2 n \ell+\sum_{i \in I} \nu\left(x_{i}-2 \ell\right)+4 \ell^{2}+4 \ell<N<(2 n+2) \ell+\sum_{i=1}^{n} \nu\left(x_{i}-2 \ell\right)-4 \ell^{3} .
$$

Proof. By (5) and (12),

$$
\nu\left(x_{i}-2 \ell\right)= \begin{cases}8 \ell^{3}+\ell, & i=1, \ldots, a \\ 8 \ell^{3}, & i=a+1, \ldots, n .\end{cases}
$$

By (14) and (10),

$$
\sum_{i=1}^{n} \nu\left(x_{i}-2 \ell\right)=a\left(8 \ell^{3}+\ell\right)+(n-a)\left(8 \ell^{3}\right)=a \ell+8 n \ell^{3}=N+4 \ell^{3}-2 n \ell,
$$


thus, the second inequality of (13) holds. On the other hand, by (14) and (15),

$$
\sum_{i \in I} \nu\left(x_{i}-2 \ell\right) \leqslant \sum_{i=1}^{n} \nu\left(x_{i}-2 \ell\right)-8 \ell^{3}=N-4 \ell^{3}-2 n \ell<N-\left(4 \ell^{2}+4 \ell\right)-2 n \ell
$$

where the last inequality holds, since $\ell \geqslant 2$. Hence, the first inequality of (13) holds too.

Let $A_{i}$ and $B_{i}, i=1, \ldots, 2 n$, be a family of $4 n$ pairwise disjoint sets with sizes:

$$
\left|A_{i}\right|=\left\{\begin{array}{l}
3 \ell-1 \text { for } i=1, \ldots, n \\
2 \ell-1 \text { for } i=n+1, \ldots, 2 n
\end{array}\right.
$$

and

$$
\left|B_{i}\right|=\left\{\begin{array}{l}
x_{i}-3 \ell+1 \text { for } i=1, \ldots, n \\
b_{i} \quad \text { for } i=n+1, \ldots, 2 n
\end{array}\right.
$$

where the $b_{i}$ 's differ from each other by at most one and are chosen in such a way that

$$
\sum_{i=1}^{2 n}\left(\left|A_{i}\right|+\left|B_{i}\right|\right)=N
$$

Observe that $b_{i}$ 's are well defined and positive. Indeed, by (16), (17), (12), and (10), using also the inequality $4 \ell n\left(\ell^{2}+\ell+1\right) \leqslant 8 \ell^{3}+2 \ell n-4 \ell^{3}$, which, due to $(11)$, is valid for $\ell \geqslant 2$,

$$
\begin{aligned}
\sum_{i=1}^{2 n}\left|A_{i}\right|+\sum_{i=1}^{n}\left|B_{i}\right| & =n(2 \ell-1)+\sum_{i=1}^{n} x_{i}=n(2 \ell-1)+a+n\left(4 \ell^{2}(\ell+1)+2 \ell\right) \\
& <a \ell+4 \ell n\left(\ell^{2}+\ell+1\right)-n \leqslant N-n .
\end{aligned}
$$

Finally, since the $b_{i}$ 's differ from each other by at most one, we have that, by the R-H-S of (13) and by (14), for $i=n+1, \ldots, 2 n$,

$$
\begin{aligned}
\left|A_{i}\right|+\left|B_{i}\right| & \leqslant\left\lceil\frac{N}{n}\right\rceil<\frac{N}{n}+1<\frac{n \cdot \max _{i} \nu\left(x_{i}-2 \ell\right)+2 n \ell}{n}+1 \\
& <\frac{n\left(8 \ell^{3}+\ell\right)+2 n \ell}{n}+1=8 \ell^{3}+3 \ell+1<10 \ell^{3} .
\end{aligned}
$$

\subsection{Main construction}

Our construction stems from a base graph $G$ which consists of a maximally non-hamiltonian graph $G_{1}$ on $n$ vertices $\{1, \ldots, n\}$ with bounded degree to which $n$ pendant vertices $\{n+1, \ldots, 2 n\}$ have been added, so that for each $i=1, \ldots, n$, the pair $\{i, n+i\}$ is an edge of $G$. By analyzing the constructions in $[2,3,4]$ one can see that the hamiltonian 
saturated graphs obtained there do have bounded maximum degree. An alternative way is by combining (1) with a result of Bondy [1] (cf. [8]).

Fix $\ell \geqslant 2$. The desired $2 \ell$-graph $H$ will be defined on an $N$-vertex set

$$
V=\bigcup_{i=1}^{2 n} U_{i}
$$

where $U_{i}=A_{i} \cup B_{i}$ and $A_{i}, B_{i}$ are given in the previous subsection. Note that, by (12), for each $i=1, \ldots, n$, we have $\left|A_{i} \cup B_{i}\right|=x_{i} \leqslant 10 \ell^{3}$. This and (19) imply together that for all $i=1, \ldots, 2 n$,

$$
\left|U_{i}\right| \leqslant 10 \ell^{3}
$$

Before defining the edge set of $H$, we need some more terminology and notation, which will be illustrated by an example. For a graph $F$ and a set $U \subset V(F)$, denote by $F[U]$ the subgraph of $F$ induced by $U$. For $S \subset V$, set

$$
\operatorname{tr}(S)=\left\{i: S \cap U_{i} \neq \emptyset\right\} \text { and } \min (S)=\min \{i \in \operatorname{tr}(S)\}
$$

(The set $\operatorname{tr}(S)$ is often called the trace of $S$, but we will not use this name here.)

Example 1. In Fig. 1 we have $\operatorname{tr}\left(e_{1}\right)=\{1,2\}, \operatorname{tr}\left(e_{2}\right)=\{1,3,2 n\}, \operatorname{tr}\left(e_{3}\right)=\{2,3\}$ and $\operatorname{tr}\left(e_{4}\right)=\{3, n+1\}$ and thus, $\min \left(e_{1}\right)=1, \min \left(e_{2}\right)=1, \min \left(e_{3}\right)=2$ and $\min \left(e_{4}\right)=3$.

Further, let $c(S)$ be the number of connected components of $G^{3}[\operatorname{tr}(S)]$, where $G^{3}$ is the third power of $G$, that is, the graph with the same vertex set as $G$, but with edges joining all pairs of distinct vertices at distance at most three in $G$.

The role of the third power can be explained as follows. In order to find a hamiltonian $(\ell, 2 \ell)$-cycle in $H+e$, we will look for a hamiltonian path between two non-adjacent vertices of $G_{1}$, selected from the vertices of $\operatorname{tr}(e)$ or their neighbors. In the worst case, $\operatorname{tr}(e) \subset\{n+1, \ldots, 2 n\}$ and we will be forced to find a hamiltonian path between the neighbors $u, v$ of some vertices $n+u$ and $n+v$. Our construction will yield $c(e) \geqslant \ell+1 \geqslant 2$ which allows us to select $n+u$ and $n+v$ so that they are non-adjacent in $G^{3}$. Consequently, $u$ and $v$ will be non-adjacent in $G_{1}$, which, by the choice of $G_{1}$, guarantees the existence (in $G_{1}$ ) of a hamiltonian path between $u$ and $v$.

We define the ultimate $2 \ell$-graph $H$ via three other hypergraphs. Let

$$
H_{1}=\left\{e \in\left(\begin{array}{c}
V \\
2 \ell
\end{array}\right): \operatorname{tr}(e) \in G \text { and }\left|A_{i} \cap e\right|=\ell \text { for both } i \in \operatorname{tr}(e)\right\} .
$$

We split $H_{1}=H_{1}^{1} \cup H_{1}^{2}$, where $H_{1}^{1}=\left\{e \in H_{1}: \operatorname{tr}(e) \in G_{1}\right\}$. Further, let

$$
H_{2}=\left\{e \in\left(\begin{array}{c}
V \\
2 \ell
\end{array}\right):\left|e \cap U_{\min (e)}\right| \geqslant \ell+1\right\} .
$$

Example 2. Recall that, in Fig.1, $\operatorname{tr}\left(e_{1}\right)=\{1,2\}$. Moreover, $\left|e \cap A_{1}\right|=\left|e \cap A_{2}\right|=3=\ell$. Thus, if $\{1,2\}$ is an edge of $G$, then $e_{1} \in H_{1}$ (more precisely, $e_{1} \in H_{1}^{1}$ ). Furthermore, $\operatorname{tr}\left(e_{2}\right)=\{1,3,2 n\}$ and $\min \left(e_{2}\right)=1$. Since $\left|e_{2} \cap U_{1}\right|=4=\ell+1$, we have $e_{2} \in H_{2}$. 
Similarly, $\left|e_{4} \cap U_{3}\right|=5 \geqslant \ell+1$, so $e_{4} \in H_{2}$ too. Finally, $\left|e_{3} \cap U_{3}\right| \geqslant \ell+1$, but $\min \left(e_{3}\right)=2$ and $\left|e_{3} \cap U_{2}\right|=1$. Hence $e_{3} \notin H_{2}$. Since $e_{3} \not \subset A_{2} \cup A_{3}, e_{3} \notin H_{1}$ either, regardless of whether $\{2,3\}$ is an edge of $G$ or not.

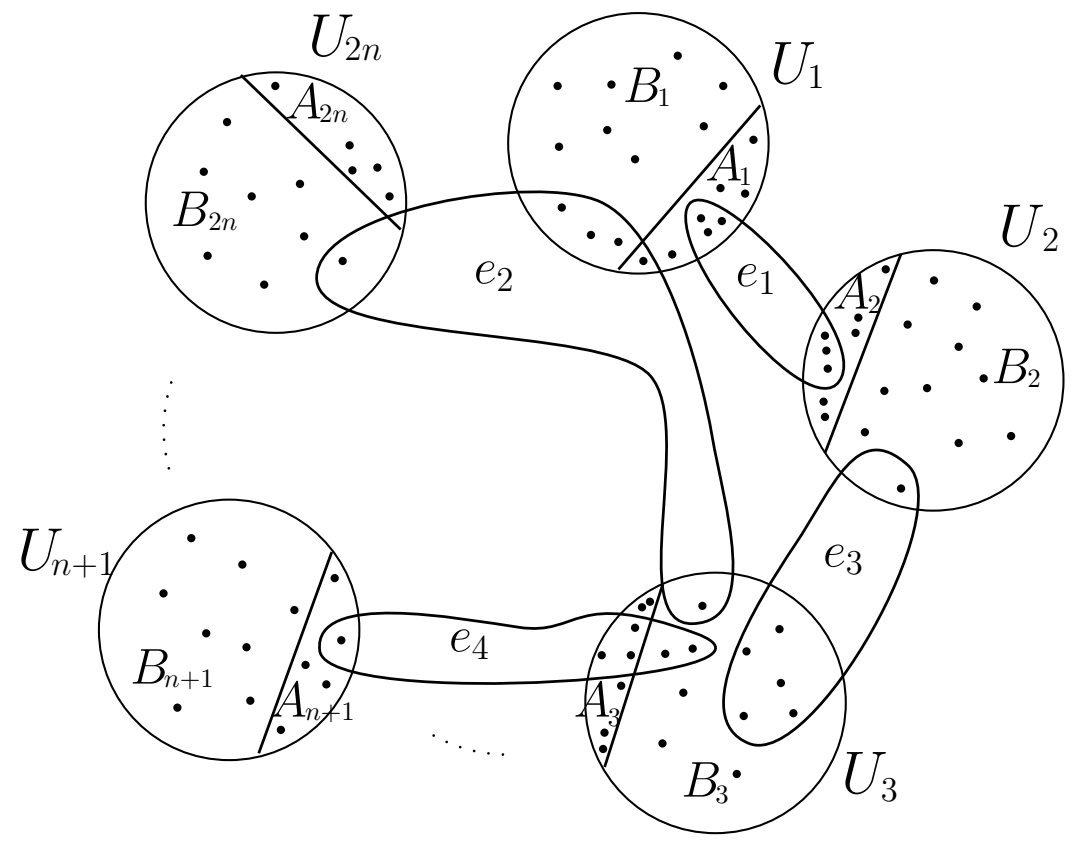

Figure 1: An illustration to construction: $\ell=3$.

Note that if $P$ is an $(\ell, 2 \ell)$-path in $H_{2}$, then there is an index $i$ such that every edge of $P$ draws at least $\ell+1$ vertices from $U_{i}$. Indeed, let $e, e^{\prime} \in P$ with $\left|e \cap e^{\prime}\right|=\ell$. Let $i=\min (e)$. Since $\left|e \cap U_{i}\right| \geqslant \ell+1,\left|e^{\prime} \cap U_{i}\right| \geqslant 1$. Hence, $i \in \operatorname{tr}\left(e^{\prime}\right)$ and so $\min \left(e^{\prime}\right) \leqslant \min (e)$. By symmetry, $\min (e) \leqslant \min \left(e^{\prime}\right)$. Thus $\min \left(e^{\prime}\right)=\min (e)=i$. By transitivity, $\min (f)=i$ for every $f \in P$.

The third element of the construction is

$$
H_{3}=\left\{e \in\left(\begin{array}{c}
V \\
2 \ell
\end{array}\right): c(e) \leqslant \ell\right\} .
$$

Note that

$$
H_{1} \cup H_{2} \subseteq H_{3},
$$

where $H_{1} \cup H_{2}$ is a $2 \ell$-graph with vertex set $V$ whose edge set is the union of the edge sets of $H_{1}$ and $H_{2}$. Indeed, if $e \in H_{1}$, then $\operatorname{tr}(e) \in G_{1}$ and so $c(e)=1 \leqslant \ell$. If $e \in H_{2}$, then $\left|e \cap U_{\min (e)}\right| \geqslant \ell+1$ and, consequently, $|\operatorname{tr}(e)| \leqslant 1+(\ell-1)=\ell$. Clearly, $c(e) \leqslant|\operatorname{tr}(e)|$, hence (21) follows.

We are going to show (cf. Lemma 5 in Section 3) that $H_{1} \cup H_{2}$ is non- $\ell$-hamiltonian. Finally, we define $H$ as a non- $\ell$-hamiltonian $2 \ell$-graph satisfying the containments

$$
H_{1} \cup H_{2} \subseteq H \subseteq H_{3}
$$


and such that $H+e$ is $\ell$-hamiltonian for every $e \in H_{3} \backslash H$. (If $H_{3}$ is non- $\ell$-hamiltonian itself, we set $H=H_{3}$.)

\subsection{Proof of Theorem 1}

In [8] we proved the following result. Let $\operatorname{comp}(F)$ denote the number of connected components of a graph $F$.

Claim 1. Let $r, \ell$, and $\Delta$ be constants. If $\Delta(G) \leqslant \Delta$, then the number of $r$-element subsets $T \subseteq V(G)$ with $\operatorname{comp}(G[T]) \leqslant \ell$ is $O\left(n^{\ell}\right)$.

Theorem 1 is an consequence of Claim 1, our construction presented in the previous subsection, and the following two lemmas the proofs of which are deferred to the later sections. Lemma 5 guarantees that the definition of $H$ is not vacuous.

Lemma 5. $H_{1} \cup H_{2}$ is non- $\ell$-hamiltonian.

Lemma 6 implies quickly that $H$ is indeed $\ell$-hamiltonian saturated (see the proof of Theorem 1 below.)

Lemma 6. For every $e \in\left(\begin{array}{c}V \\ 2 \ell\end{array}\right) \backslash H_{3}$, the $2 \ell$-graph $H_{1} \cup H_{2}+e$ is $\ell$-hamiltonian.

Proof of Theorem 1. By $(2), \operatorname{sat}(N, 2 \ell, \ell)=\Omega\left(N^{\ell}\right)$. In order to prove the upper bound, we begin by showing that $|H|=O\left(N^{\ell}\right)$. Observe that

$$
H_{3}=\bigcup_{T \subset V(G)}\left\{e \in\left(\begin{array}{c}
V \\
2 \ell
\end{array}\right): \operatorname{tr}(e)=T\right\}
$$

where the sum is over all subsets $T$ of $V(G)$ of size at most $2 \ell$ with $\operatorname{comp}\left(G^{3}[T]\right) \leqslant \ell$. Since $G_{1}$ has bounded degree, so does $G$ and $G^{3}$. Thus, by Claim 1 with $r \leqslant 2 \ell$, the number of such subsets $T$ is $O\left(n^{\ell}\right)$. Moreover, given $T$,

$$
\left|\left\{e \in\left(\begin{array}{c}
V \\
2 \ell
\end{array}\right): \operatorname{tr}(e)=T\right\}\right| \leqslant\left(\begin{array}{c}
\sum_{i \in T}\left|U_{i}\right| \\
2 \ell
\end{array}\right) \leqslant\left(|T| \cdot 10 \ell^{3}\right)^{2 \ell}=O(1),
$$

by (20). Consequently, $\left|H_{3}\right|=O\left(n^{\ell}\right)=O\left(N^{\ell}\right)$ and, thus, also $|H|=O\left(N^{\ell}\right)$.

It remains to show that $H$ is $\ell$-hamiltonian saturated. Recall that, by construction (and Lemma 5), $H$ is non- $\ell$-hamiltonian. Let $e \in\left(\begin{array}{c}V \\ 2 \ell\end{array}\right) \backslash H$. If $e \in H_{3}$ then, by the definition of $H, H+e$ is $\ell$-hamiltonian. On the other hand, if $e \in\left(\begin{array}{c}V \\ 2 \ell\end{array}\right) \backslash H_{3}$, then $H+e \supseteq H_{1} \cup H_{2}+e$ is $\ell$-hamiltonian by Lemma 6 . This shows that $H$ is, indeed, $\ell$-hamiltonian saturated and thus, the proof of Theorem 1 is completed.

\section{Proof of Lemma 5.}

\section{$3.1(\ell, 2 \ell)$-paths in $H_{1} \cup H_{2}$}

Before turning to the actual proof, we first prove a result about $(\ell, 2 \ell)$-paths in $H_{1} \cup H_{2}$. 
Proposition 7. Let $m \geqslant 1$ and $P=\left(e, e_{1}, \ldots, e_{m}, e^{\prime}\right)$ be an $(\ell, 2 \ell)$-path in $H_{1} \cup H_{2}$ such that $e, e^{\prime} \in H_{1}^{1}$ and $e_{i} \in H_{1}^{2} \cup H_{2}, i=1, \ldots, m$. The following hold:

(a) $P$ does not contain an edge $f \in H_{1}^{2}$ disjoint from $e \cup e^{\prime}$;

(b) $P$ does not contain two disjoint edges $f, f^{\prime} \in H_{1}^{2}$;

(c) $\min \left(e_{i}\right) \in \operatorname{tr}(e) \cap \operatorname{tr}\left(e^{\prime}\right), i=1, \ldots, m$.

In the proof of Proposition 7, we will need the following result.

Claim 2. Let $m \geqslant 1$ and let $P=\left(e, e_{1}, \ldots, e_{m}, e^{\prime}\right)$ be an $(\ell, 2 \ell)$-path such that $e, e^{\prime} \in H_{1}$ and $e_{i} \in H_{2}, i=1, \ldots, m$. Then $\min \left(e_{1}\right)=\cdots=\min \left(e_{m}\right) \in \operatorname{tr}(e) \cap \operatorname{tr}\left(e^{\prime}\right), i=1, \ldots, m$.

Proof. Let $\alpha=\min \left(e_{1}\right)$. Then, by the definition of $H_{2}$ and the fact that $\left|e_{1} \backslash e_{2}\right|=\ell<\ell+1$, we have $\alpha \in \operatorname{tr}\left(e_{2}\right)$. Hence, $\min \left(e_{2}\right) \leqslant \alpha=\min \left(e_{1}\right)$. By symmetry, $\min \left(e_{1}\right) \leqslant \min \left(e_{2}\right)$. Thus, $\min \left(e_{1}\right)=\min \left(e_{2}\right)$. By transitivity, $\min \left(e_{i}\right)=\alpha$ for every $i=1, \ldots, m$. By the same token, $\alpha \in \operatorname{tr}(e)$ and $\alpha \in \operatorname{tr}\left(e^{\prime}\right)$.

Proof of Proposition \%. Since $m \geqslant 1$, we have $e \cap e^{\prime}=\emptyset$. If $P$ does not contain any edge of $H_{1}^{2}$, then the statements (a) and (b) are vacuous, while (c) follows from Claim 2. Assume that $H_{1}^{2} \cap P=\left\{f_{1}, \ldots, f_{t}\right\}$ where $t \geqslant 1$ and $f_{j}, j=1, \ldots, t$, are listed in order of appearance on $P$. Let $\operatorname{tr}\left(f_{1}\right)=\{\alpha, n+\alpha\}$. Furthermore, let $f_{0}=e$ and $f_{t+1}=e^{\prime}$.

If $f_{j} \cap f_{j+1} \neq \emptyset$ then, trivially,

$$
\operatorname{tr}\left(f_{j}\right) \cap \operatorname{tr}\left(f_{j+1}\right) \neq \emptyset \quad j=0,1, \ldots, t .
$$

Otherwise, (22) holds by Claim 2. It follows by the structure of $G$ that $\operatorname{tr}\left(f_{j}\right)=\{\alpha, n+\alpha\}$, $j=1, \ldots, t$, and $\alpha \in \operatorname{tr}\left(f_{j}\right), j \in\{0, t+1\}$, that is, $\alpha \in \operatorname{tr}(e) \cap \operatorname{tr}\left(e^{\prime}\right)$.

Since $e \cap e^{\prime}=\emptyset$ and $\left|A_{\alpha} \cap f_{j}\right|=\ell$ for every $j \in\{0, \ldots, t+1\}$, (a) holds by the first part of (16), while (b) holds by the second part of (16). Note that it follows that (c) holds for every edge $f_{j}, j=1, \ldots, t$, that is, for every edge $e_{i} \in H_{1}^{2}$.

Let us now consider $e^{\prime \prime} \in P \cap H_{2}$. If $m \geqslant 3$, then, by (a) and (b), the only edge in $\left\{e_{1}, \ldots, e_{m}\right\} \cap H_{1}^{2}$ is either $\left\{e_{1}\right\}$ or $\left\{e_{m}\right\}$. Without loss of generality assume that $e_{1} \in H_{1}^{2}$ and $e_{m} \in H_{2}$. (For $m=2$, we may assume the same with $e^{\prime \prime}=e_{m}$.) By Claim 2 applied to the path from $e_{1}$ to $e^{\prime}$, we conclude that $\min \left(e^{\prime \prime}\right) \in \operatorname{tr}\left(e_{1}\right)=\{\alpha, n+\alpha\}$, as well as, $\min \left(e^{\prime \prime}\right) \in \operatorname{tr}\left(e^{\prime}\right) \subset\{1, \ldots, n\}$. Hence, $\min \left(e^{\prime \prime}\right)=\alpha \in \operatorname{tr}(e) \cap \operatorname{tr}\left(e^{\prime}\right)$ and (c) holds.

\subsection{Proof of Lemma 5.}

In this subsection we complete the proof of Lemma 5 .

Proof of Lemma 5. Suppose $C$ is a hamiltonian $(\ell, 2 \ell)$-cycle in $H_{1} \cup H_{2}$. We are going show that $|V(C)|<N$ which will be a contradiction. Our proof at some point (cf. proof of Claim 4) relies on the assumption that the graph $G_{1}$ is not hamiltonian. Let $M=\left\{e_{1}, \ldots, e_{m}\right\}$ be a maximal set of pairwise disjoint edges of $C \cap H_{1}^{1}$, listed in the 
order of appearance on $C$. Further, for $i=1, \ldots, m$, let $P_{i}$ be the $(\ell, 2 \ell)$-path in $C$ joining the last $\ell$ vertices of $e_{i}$ with the first $\ell$ vertices of $e_{i+1}$, where $e_{m+1}:=e_{1}$. Notice that

$$
C \backslash M=\bigcup_{i=1}^{m} P_{i},
$$

where all $P_{i}^{\prime}$ 's are vertex disjoint, see Fig. 2.

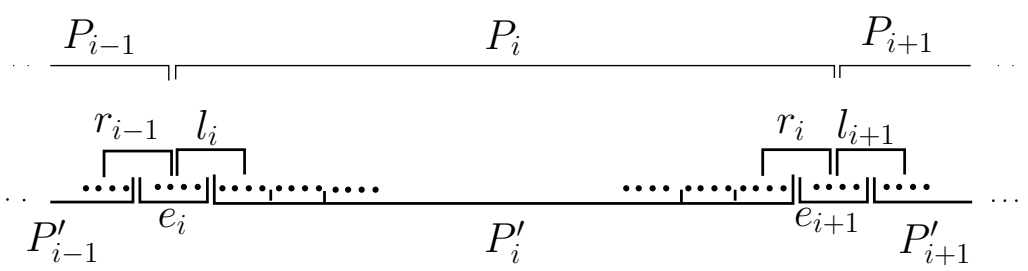

Figure 2: Fragment of $C$

Let $l_{i}$ be the first edge of $P_{i}$ and $r_{i}$ be the last edge of $P_{i}$ (note that they may coincide). We also define $P_{i}^{\prime}$ to be the $(\ell, 2 \ell)$-path arising from $P_{i}$ by removing both $l_{i}$ and $r_{i}$. Note that, by the definition of $M$,

$$
P_{i}^{\prime} \subset H_{1}^{2} \cup H_{2}
$$

We call $P_{i}^{\prime}$ trivial if $P_{i}^{\prime} \subset H_{1}^{2}$. We further define

$$
P_{i}^{\prime \prime}=P_{i}^{\prime} \cap H_{2}
$$

Note that $P_{i}^{\prime \prime}$ is an $(\ell, 2 \ell)$-path, too. Indeed, by Proposition 7a), every edge in $P_{i}^{\prime} \cap H_{1}^{2}$ intersects $l_{i}$ or $r_{i}$ (and thus, is the first or the last edge of $P_{i}^{\prime}$ ).

If $P_{i}^{\prime \prime}$ is non-empty, then let

$$
\alpha_{i}=\min (f) \text { for every } f \in P_{i}^{\prime \prime}
$$

By Claim 2, $\alpha_{i}$ is well defined.

Observe that each edge $e \in\left(H_{1}^{1} \cap C\right) \backslash M$ intersects some $e_{i} \in M$, so $e=l_{i}$ or $e=r_{i-1}$. We call an edge $l_{i}$ (or $r_{i}$ ) bad if it belongs to $H_{1}^{1},\left|P_{i}\right| \geqslant 2$, and $\operatorname{tr}\left(l_{i}\right) \neq \operatorname{tr}\left(e_{i}\right)$ $\left(\operatorname{tr}\left(r_{i}\right) \neq \operatorname{tr}\left(e_{i+1}\right)\right.$, resp.). We call $P_{i}$ problematic if either $l_{i}$ or $r_{i}$ is bad or $P_{i}^{\prime}$ contains an edge from $H_{1}^{2}$. Otherwise, we call $P_{i}$ nice.

Let $\operatorname{Tr}(M)=\{\operatorname{tr}(e): e \in M\}$ be a graph defined by the traces of edges in $M$. Clearly, $|\operatorname{Tr}(M)|=m$. Since, for each $e \in M$ and $j \in \operatorname{tr}(e),\left|e \cap A_{j}\right|=\ell$,

$$
\Delta(\operatorname{Tr}(M)) \leqslant 2
$$


by (16). In particular

$$
m \leqslant n \text {. }
$$

We need, however, better bounds on $m$. Let $q$ be the number of problematic $(\ell, 2 \ell)$-paths among $P_{1}, \ldots, P_{m}$.

Claim 3.

$$
m \leqslant\left\lfloor n-\frac{q}{2}\right\rfloor
$$

Proof. Let $P$ be problematic. Suppose $e$ is a bad edge in $P$. If $e=l_{i}$ then since $\operatorname{tr}\left(l_{i}\right) \neq$ $\operatorname{tr}\left(e_{i}\right)$, there exists $\beta \in \operatorname{tr}(e)$ such that $\left|\left(e \cap A_{\beta}\right) \backslash e_{i}\right|=\ell$. Since $|P| \geqslant 2$,

$$
\left|\left(e \cap A_{\beta}\right) \backslash\left(e_{i} \cup e_{i+1}\right)\right|=\ell,
$$

as well. By symmetry, the same holds if $e=r_{i}$. If $P^{\prime}$ contains an edge $e$ which belongs to $H_{1}^{2}$, then (30) is also true, since $e$ does not intersect any edge of $M$. To sum up, for each $i=1, \ldots, m$, there exists $\beta_{i} \in \operatorname{tr}\left(P_{i}\right)$ such that

$$
\left|\left(V\left(P_{i}\right) \cap A_{\beta_{i}}\right) \backslash \bigcup_{j=1}^{m} e_{j}\right| \geqslant\left\{\begin{array}{l}
\ell \text { if } P_{i} \text { is problematic } \\
0 \text { otherwise. }
\end{array}\right.
$$

Note that $\beta_{i}$ 's need not be different. Since $\left|A_{\beta_{i}}\right| \leqslant 3 \ell-1,(31)$ implies that $\operatorname{deg}_{\operatorname{Tr}(M)}\left(\beta_{i}\right) \leqslant 1$ if $P_{i}$ is problematic (and $\operatorname{deg}_{\operatorname{Tr}(M)}\left(\beta_{i}\right) \leqslant 2$ if not). If two problematic $P_{i}$ 's yield the same $\beta_{i}$ as above, then we conclude that $\operatorname{deg}_{\operatorname{Tr}(M)}\left(\beta_{i}\right)=0$. Thus,

$$
\sum_{i=1}^{n} \operatorname{deg}_{\operatorname{Tr}(M)}\left(\beta_{i}\right) \leqslant 2 n-q \text {. }
$$

Therefore,

$$
m=|\operatorname{Tr}(M)| \leqslant\left\lfloor\frac{2 n-q}{2}\right\rfloor .
$$

Claim 4. Suppose that $P_{i}^{\prime} \neq \emptyset$ for every $i=1, \ldots, m$. Then

$$
m \leqslant n-1
$$

Proof. If $q \geqslant 1$, then the claim follows by Claim 3. Assume that $q=0$ and $|\operatorname{Tr}(M)|=$ $m=n$. Then, by (27), $\operatorname{Tr}(M)$ is a 2-regular spanning subgraph of $G_{1}$. Since $q=0$, each $P_{i}$ is nice and so

$$
P_{i}^{\prime} \subset H_{2},
$$

by (24). Let $f_{i}$ be any edge of $P_{i}^{\prime}$. Recall that $\alpha_{i}=\min \left(f_{i}\right)$, see $(26)$ and because $P_{i}^{\prime}=P_{i}^{\prime \prime}$ by (33). If $l_{i} \in H_{1}^{2} \cup H_{2}$, then $\alpha_{i} \in \operatorname{tr}\left(e_{i}\right)$ by Proposition 7(c) applied to $P+e_{i}+e_{i+1}$. Otherwise, if $l_{i} \in H_{1}^{1}$, then $\alpha_{i} \in \operatorname{tr}\left(l_{i}\right)$, again by Proposition $7(\mathrm{c})$, this time applied to $P_{i}$. Since $P_{i}$ is nice, $l_{i}$ is not bad and so, $\operatorname{tr}\left(e_{i}\right)=\operatorname{tr}\left(l_{i}\right)$. Hence, $\alpha_{i} \in \operatorname{tr}\left(e_{i}\right)$, as before. By symmetry, $\alpha_{i} \in \operatorname{tr}\left(e_{i+1}\right)$, too. Thus, $\operatorname{Tr}(M)$ is connected and, consequently, $\operatorname{Tr}(M)$ is a hamiltonian cycle in $G_{1}$, a contradiction. 
Claim 5. If $P_{i}$ is nice, then

$$
\left|V\left(P_{i}^{\prime}\right)\right| \leqslant \nu\left(x_{\alpha_{i}}-2 \ell\right) .
$$

Proof. Since $P_{i}$ is nice, $P_{i}^{\prime}=P_{i}^{\prime \prime} \subset H_{2}$ by (24). If $P_{i}^{\prime}=\emptyset$, then the claim trivially holds. Assume that $f_{i} \in P_{i}^{\prime}$. Then $\alpha_{i}=\min \left(f_{i}\right)$. Similarly, as in the proof of Claim 4, we infer that $\alpha_{i} \in \operatorname{tr}\left(e_{i}\right)$ and $\alpha_{i} \in \operatorname{tr}\left(e_{i+1}\right)$. In particular, since $e_{i}, e_{i+1} \in H_{1}^{1}, \alpha_{i} \leqslant n$. Thus, $\left|A_{\alpha_{i}} \cap e_{i}\right|=\ell$ and $\left|A_{\alpha_{i}} \cap e_{i+1}\right| \geqslant \ell$, which implies that $\left|V\left(P_{i}^{\prime}\right) \cap U_{\alpha_{i}}\right| \leqslant x_{\alpha_{i}}-2 \ell$. Therefore, the claim follows by the definitions of $H_{2}$ and $\nu$.

Claim 6. If $P_{i}$ is problematic, then

$$
\left|V\left(P_{i}^{\prime}\right)\right| \leqslant \nu\left(x_{\alpha_{i}}\right)+\ell
$$

Proof. By Proposition 7(a),(b) and by the choice of $M, P_{i}^{\prime}$ contains at most one edge, say $f_{i}$, from $H_{1}^{2}$. Moreover, this edge is the first or the last edge of $P_{i}^{\prime}$. The rest of $P_{i}^{\prime}$ (i.e., $P_{i}^{\prime}$ minus the first or the last $\ell$ vertices) is contained in $H_{2}$. Hence, by Claim $2, \alpha_{i} \in \operatorname{tr}\left(e_{i}\right)$ or $\alpha_{i} \in \operatorname{tr}\left(e_{i+1}\right)$. In particular, $\alpha_{i} \leqslant n$. Thus, the claim follows by the definition of $\nu$.

We are now in the position to finish the proof of Lemma 5. Suppose that there are exactly $q$ problematic paths among the $P_{i}$ 's. Let $I^{\prime} \subset[1, n]$ be the set of those indices $i$ for which $P_{i}$ is problematic, and $I^{\prime \prime}=[1, m] \backslash I^{\prime}$. By (23), Claims 5 and 6 , and Proposition 3 (applied $2 \ell$ times),

$$
\begin{aligned}
|V(C)| & =2 m \ell+\sum_{i=1}^{m}\left|V\left(P_{i}^{\prime}\right)\right| \\
& \leqslant 2 m \ell+\sum_{i \in I^{\prime}}\left(\nu\left(x_{\alpha_{i}}\right)+\ell\right)+\sum_{i \in I^{\prime \prime}} \nu\left(x_{\alpha_{i}}-2 \ell\right) \\
& \leqslant 2 m \ell+\sum_{i \in I^{\prime}}\left(\nu\left(x_{\alpha_{i}}-2 \ell\right)+2 \ell^{2}+\ell\right)+\sum_{i \in I^{\prime \prime}} \nu\left(x_{\alpha_{i}}-2 \ell\right) \\
& =2 m \ell+\sum_{i=1}^{m} \nu\left(x_{\alpha_{i}}-2 \ell\right)+\left(2 \ell^{2}+\ell\right) q .
\end{aligned}
$$

If $q \geqslant 1$, then ( $\operatorname{since} \nu\left(x_{\alpha_{i}}-2 \ell\right) \geqslant x_{\alpha_{i}}-2 \ell>4 \ell^{2}+2 \ell$ ) the maximum is attained for $m=n-1$ and $q=2$, by Claim 3. Hence,

$$
|V(C)| \leqslant 2 n \ell+\sum_{i \in I} \nu\left(x_{\alpha_{i}}-2 \ell\right)+2\left(2 \ell^{2}+\ell\right)
$$

where $I \subset[1, n]$ with $|I| \leqslant n-1$. Otherwise, by Claim 4 , either $m \leqslant n-1$ or $m \leqslant n$ and $P_{i}^{\prime}=\emptyset$ for some $i \in\{1, \ldots, m\}$. In both these cases (34) holds as well. Therefore, by (13), $|V(C)|<N$, and so $C$ cannot be a hamiltonian $(\ell, 2 \ell)$-cycle, a contradiction. 


\section{Proof of Lemma 6.}

\subsection{The idea of the proof}

One can easily construct $n$ disjoint $(\ell, 2 \ell)$-paths $P_{1}, \ldots P_{n}$ in $H_{2}$. Each such path $P_{j}$, however, is relatively short. Indeed, recall that by the definition of $H_{2}$, every edge of $P_{j}$ draws at least $\ell+1$ vertices from some fixed set $U_{i_{j}}$.

Edges from $H_{1}$ will serve as bridges joining the paths $P_{j}$. We have seen in the proof of Lemma 5 that, since $G_{1}$ is not Hamiltonian, we can use at most $n-1$ bridges. Fortunately, the new edge $e \notin H$ will play the role of an additional bridge in $H$, that, together with original $n-1$ edges of $M$, will 'glue' all paths $P_{1}, \ldots, P_{n}$ into a hamiltonian $(\ell, 2 \ell)$-cycle in $H$.

The use of $H_{3}$ is crucial for the argument. It allows us, when proving the existence of a hamiltonian $(\ell, 2 \ell)$-cycle in $H+e$, to restrict only to $e \in\left(\begin{array}{c}V \\ 2 \ell\end{array}\right) \backslash H_{3}$, for which we know that $c(e) \geqslant \ell+1$. The remaining edges (i.e. those in $H_{3} \backslash H$ ), which are relatively rare but cumbersome, can be ignored just by the definition of $H$.

\subsection{Proof of Lemma 6}

The forthcoming proof will be illustrated by some diagrams in which we apply the following notation.

- $I$ denotes a vertex from $A_{i}$

- $I, I, \ldots, I$ denotes a sequence of different vertices from $A_{i}$

- $i$ denotes a vertex from $U_{i}$ (we do not exclude $A_{i}$ )

- $i, i, \ldots, i$ denotes a sequence of different vertices from $U_{i}$

- * denotes a vertex from $V$

- $*, *, \ldots, *$ denotes a sequence of different vertices from $V$

Proof of Lemma 6. Let $e \in\left(\begin{array}{c}V \\ 2 \ell\end{array}\right) \backslash H_{3}$. Recall that, by the definition of $H_{3}, c(e) \geqslant \ell+1$. For a subset $Z \subseteq \operatorname{tr}(e)$ let $e(Z)=\{u \in e: \operatorname{tr}(u) \in Z\}$. Let $X$ be the vertex set of the component of $G^{3}[\operatorname{tr}(e)]$ which contains vertex $i=\min (e)$ and let $Y=\operatorname{tr}(e) \backslash X$. Note that, since $c(e) \geqslant \ell+1$,

$$
|e(X)| \leqslant \ell
$$

If for some $s \in Y$ we have $\left|e \cap U_{s}\right| \geqslant \ell$, then let $j=s$. Otherwise, let $j=\min (e(Y))$. By the choice of $j$

$$
\left|U_{t} \cap e\right| \leqslant \ell-1 \text { for all } t \notin\{i, j\} \text {. }
$$


Also, as $i$ and $j$ are in different components of $G^{3}[\operatorname{tr}(e)]$, they do not form an edge of $G$. Even more, if $i=n+i^{\prime}$ or $j=n+j^{\prime}$ for some $1 \leqslant i^{\prime}, j^{\prime} \leqslant n$, then, as $i$ and $j$ are in different components of $G^{3}[\operatorname{tr}(e)]$, we have $i j^{\prime}, i^{\prime} j, i^{\prime} j^{\prime} \notin G_{1}$ either.

Suppose first that $i, j \in\{1, \ldots, n\}$. Let $P_{0}$ be a 3-edge $(\ell, 2 \ell)$-path with the edge $e$ in the middle and two edges $e^{\prime}$ and $e^{\prime \prime}$ from $H_{2}$. The first $\ell$ vertices of $e$ belong to $e(Y)$ and the first one of them must be from $U_{j}$. The last $\ell$ vertices of $e$ contain $e(X)$ and the last of them must be from $U_{i}$. The first edge of $P_{0}, e^{\prime}$, begins with $\ell$ vertices of $U_{j}$, the last (third) edge of $P_{0}, e^{\prime \prime}$, ends with $\ell$ vertices of $U_{i}$ (see the diagram below).

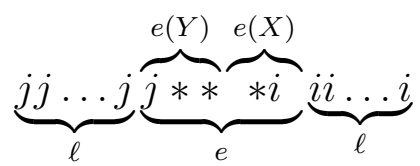

Due to this deliberate construction and the choice of $j$, we have $\min \left(e^{\prime}\right)=j$ and $\left|e^{\prime} \cap U_{j}\right| \geqslant$ $\ell+1$, so that indeed $e^{\prime} \in H_{2}$. Similarly, $e^{\prime \prime} \in H_{2}$. As observed above, ij $\notin G_{1}$.

If $i=n+i^{\prime}$ and $j=n+j^{\prime}$, then $P_{0}$ is, if possible, of the form

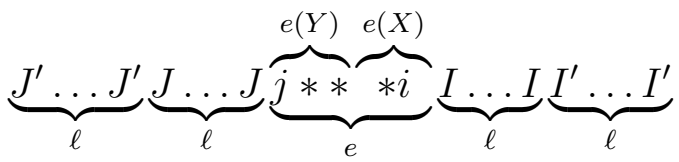

In this case the first and the last edge of $P_{0}$ belong to $H_{1}^{2}$, and the second and the penultimate - to $H_{2}$. However, by (16), this construction is not feasible if $\left|e \cap A_{i}\right|=\ell$ or $\left|e \cap A_{j}\right|=\ell$. In such cases we modify $P_{0}$ as follows (let, say, $\left|e \cap A_{i}\right|=\ell$ )

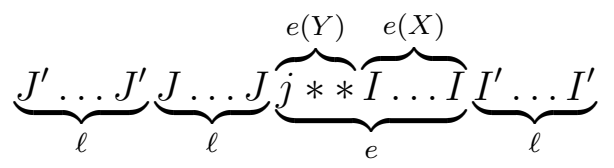

As observed above, $i^{\prime} j^{\prime} \notin G_{1}$. If $i \leqslant n$ and $j=n+j^{\prime}$, then the right-hand side of $P_{0}$ is like in diagram (37), while the left-hand side is like in diagram (38) or (39). The construction for $i=n+i^{\prime}$ and $j \leqslant n$ is analogous.

Since $G_{1}$ is maximally non-hamiltonian, it contains a hamiltonian path $v_{1} v_{2} \cdots v_{n-1} v_{n}$, where $v_{1} \in\left\{i, i^{\prime}\right\}$ and $v_{n} \in\left\{j, j^{\prime}\right\}$, depending on the case. Based on this hamiltonian path, we are building a hamiltonian $(\ell, 2 \ell)$-cycle in $H$ as follows.

Note that by (35) and by the construction of $P_{0}$

$$
\left|U_{t} \cap P_{0}\right| \leqslant\left\{\begin{array}{l}
2 \ell-1 \text { for } t \in\left\{v_{1}, v_{n}\right\} \\
\ell-1 \text { for } t \in\left\{v_{2}, \ldots, v_{n-1}\right\} .
\end{array}\right.
$$

First, we construct $n-1$ pairwise disjoint edges, $e_{1} \ldots, e_{n-1} \in H_{1}$, such that they are also disjoint from $e$ and for each $t=1, \ldots, n-1, e_{t}$ contains $\ell$ vertices from $A_{v_{t}}$ followed by $\ell$ vertices from $A_{v_{t+1}}$ (see the diagram below)

$$
\underbrace{V_{t}, \ldots, V_{t}}_{\ell} \underbrace{V_{t+1}, \ldots, V_{t+1}}_{\ell} \text {. }
$$


By (40) and (16), this construction is possible.

Next, we construct $n(\ell, 2 \ell)$-paths $P_{t} \subseteq H_{2}, t=1, \ldots, n$, such that $P_{t}$ consists of all vertices from $U_{v_{t}} \backslash\left(V\left(P_{0}\right) \cup \bigcup_{t=1}^{n-1} e_{t}\right)$ and some vertices from $\bigcup_{j=n+1}^{2 n} U_{j}$, and $\left|V\left(P_{t}\right)\right|$ is as large as possible. We will do it in two stages. First, instead of $\bigcup_{j=n+1}^{2 n} U_{j}$ we use vertices from some (abstract) infinite set $B$ and denote the resulting $(\ell, 2 \ell)$-paths by $P_{t}^{\prime}$.

Recall that $\left|U_{i}\right|=x_{i}$ and that the set $e_{i-1} \cup e_{i}$ contains already $2 \ell$ vertices of $U_{i}$. Hence, if $U_{v_{i}} \cap e=\emptyset$, then we still have to use $x-2 \ell$ vertices from $U_{v_{i}}$ and so, recalling the definition of $\nu\left(x_{i}\right)$,

$$
\left|V\left(P_{i}^{\prime}\right)\right|=\nu\left(x_{i}-2 \ell\right)
$$

Otherwise, quite roughly,

$$
\left|V\left(P_{i}^{\prime}\right)\right| \geqslant \nu\left(x_{i}-4 \ell\right) \geqslant \nu\left(x_{i}-2 \ell\right)-2 \ell^{2},
$$

by Proposition 3. Note that since $|e| \leqslant 2 \ell$, $e$ intersects at most $2 \ell$ sets $U_{t}$. Bearing this in mind, we now estimate from below the total number $N^{\prime}$ of vertices appearing in all so far constructed elements:

$$
\begin{aligned}
N^{\prime} & =\left|P_{0}\right|+\sum_{t=1}^{n-1}\left|e_{t}\right|+\sum_{t=1}^{n}\left|P_{t}^{\prime}\right| \\
& \geqslant 2(n+1) \ell+\sum_{t, U_{t} \cap e=\emptyset} \nu\left(x_{t}-2 \ell\right)+\sum_{t, U_{t} \cap e \neq \emptyset}\left(\nu\left(x_{t}-2 \ell\right)-2 \ell^{2}\right) \\
& \geqslant 2(n+1) \ell+\sum_{t=1}^{n} \nu\left(x_{t}-2 \ell\right)-4 \ell^{3}>N
\end{aligned}
$$

where the last inequality holds by (13). Note that both, $N^{\prime}$ and $N$, are divisible by $\ell$. We remove $N^{\prime}-N$ vertices of $B$ from the paths $P_{1}^{\prime}, \ldots, P_{t}^{\prime}$ in such a way that each path $P_{t}^{\prime}$ gets shorter by a multiple of $\ell$ vertices and the vertices removed from each $P_{t}^{\prime}$ are the first vertices of $V\left(P_{t}^{\prime} \cap B\right)$ according to the order of appearance on $P_{t}^{\prime}$. Treating the remaining vertices as consecutive, we thus obtain a collection of paths $P_{t}^{\prime \prime}$ such that each edge of $P_{t}^{\prime \prime}$ still has at least $\ell+1$ vertices of $U_{v_{t}}$. Now we arbitrarily replace the remaining vertices of $B$ by the vertices of $\bigcup_{j=n+1}^{2 n} U_{j}$, obtaining the desired paths $P_{t} \in H_{2}$.

Finally, note that the sequence

$$
S=P_{0}, P_{1}, e_{1}, P_{2}, e_{2}, P_{3}, \ldots, e_{n-1}, P_{n}
$$

spans a hamiltonian $(\ell, 2 \ell)$-cycle in $H_{1} \cup H_{2}+e$. Indeed, the last $\ell$ vertices of $P_{0}$ and the first $\ell$ vertices of $P_{1}$ together contain at least $\ell+1$ vertices of $U_{v_{1}}$ and thus they form an edge of $H_{2}$. So do the last $\ell$ vertices of $P_{1}$ and the first $\ell$ vertices of $e_{1}$, etc. Finally, the last $\ell$ vertices of $P_{n}$ and the first $\ell$ vertices of $P_{0}$ together contain at least $\ell+1$ vertices of $U_{v_{n}}$ and so they also form an edge of $H_{2}$. 


\section{Acknowledgements}

We are extremely grateful to an anonymous referee for their careful reading of the manuscript and valuable and insightful comments leading ultimately to a much better version of the paper than originally submitted.

\section{References}

[1] J. A. Bondy. Variations on the hamiltonian theme. Canad. Math. Bull., 15:57-62, 1972.

[2] L. Clark and R. Entringer, Smallest maximally non-hamiltonian graphs, Period. Math. Hungar. 14(1), 1983, 57-68.

[3] L. Clark, R. Crane, R. Entringer and H. Shapiro. On smallest maximally nonhamiltonian graphs. In Proceedings of the seventeenth Southeastern international conference on combinatorics, graph theory, and computing (Boca Raton, Fla., 1986), 53:215-220, 1986.

[4] L. H. Clark, R. C. Entringer, H. D. Shapiro. Smallest maximally non-hamiltonian graphs II. Graphs Combin., 8:225-231, 1992.

[5] R. Glebov, Y. Person and W. Weps, On extremal hypergraphs for hamiltonian cycles. European J. Combin., 33:544-555, 2012.

[6] G. Y. Katona, Hamiltonian chains in hypergraphs, A survey. Graphs, Combinatorics, Algorithms and its Applications, (ed. S. Arumugam, B. D. Acharya, S. B. Rao), Narosa Publishing House 2004.

[7] G. Y. Katona and H. Kierstead, Hamiltonian chains in hypergraphs. J. Graph Theory, 30:205-212, 1999.

[8] A. Ruciński and A. Żak, Hamilton saturated hypergraphs of essentially minimum size, Electron. J. Combin., 20(2), 2013, \#P25.

[9] A. Ruciński and A. Żak, Upper Bounds on the Minimum Size of Hamilton Saturated Hypergraphs, Electron. J. Comb., 23(4), 2016, \#P4.12

[10] A. Żak, Growth order for the size of smallest hamiltonian chain saturated uniform hypergraphs. European J. Combin., 34:724-735, 2013. 